\title{
A SINGULAR LIMIT PROBLEM FOR A LINEAR VOLTERRA EQUATION*
}

\author{
By \\ RICHARD NOREN
}

Old Dominion University, Norfolk, Virginia

Abstract. We study the dependence on $c_{1}$ and $c_{2}$ of the solution $u\left(t, c_{1}, c_{2}\right)$ of the equation

$$
u^{\prime}(t)+\int_{0}^{t} A\left(t-s, c_{1}, c_{2}\right) u(s) d s=0, \quad u(0)=1
$$

where the conditions on $A$ are stated in terms of its Fourier transform. We obtain sufficient conditions and (weaker) necessary conditions for

$$
\int_{0}^{\infty} \sup _{0 \leq c_{i} \leq 1}\left|u\left(t, c_{1}, c_{2}\right)\right| d t<\infty, \quad i=1,2
$$

and for

$$
\int_{0}^{\infty} \sup _{0 \leq c_{1}, c_{2} \leq 1}\left|u\left(t, c_{1}, c_{2}\right)\right| d t<\infty .
$$

The kernel $A$ is a combination of nonnegative nonincreasing convex functions and arises in the linear theory of viscoelastic rods and plates.

1. Introduction. We study the solution $u=u\left(t, c_{1}, c_{2}\right)$ of the scalar equation

$$
u^{\prime}(t)+\int_{0}^{t} A\left(t-s, c_{1}, c_{2}\right) u(s) d s=0, \quad t \geq 0, u(0)=1,
$$

(' indicates differentiation) where the parameters $c_{1}$ and $c_{2}$ satisfy $0 \leq c_{1} \leq 1,0 \leq$ $c_{2} \leq 1$, and the hypotheses on $A$ are stated in terms of its Fourier transform $\hat{A}$; the fact that $A$ is locally absolutely continuous follows from [3, Theorem 1.1 (i)] under the assumptions (1.2)-(1.5) below.

In this paper, the Fourier transform $\hat{h}$ is defined for a function $h$ such that $h(t) e^{-\sigma t} \in L^{1}(0, \infty)$ for all $\sigma>0$ by the formula

$$
\hat{h}(\tau)=\int_{0}^{\infty} e^{-i \tau t} h(t) d t \quad(\operatorname{Im} \tau<0), \quad \hat{h}\left(\tau_{0}\right)=\lim _{\tau \rightarrow \tau_{0}, \operatorname{Im} \tau<0} \hat{h}(\tau) \quad\left(\tau_{0} \in \mathbf{R}\right)
$$

wherever the limit exists. We suppose that $A$ satisfies

$$
\hat{A}(\tau)=\left(\hat{a}(\tau)-c_{1} i \tau^{-1}\right) f(\tau)
$$

*Received May 5, 1987. 
where

$$
\begin{gathered}
f(\tau)=f\left(\tau, c_{1}, c_{2}\right)=F\left(\left(\hat{b}(\tau)-c_{2} i \tau^{-1}\right) /\left(\hat{a}(\tau)-c_{1} i \tau^{-1}\right)\right), \\
F(w)=\frac{w+m}{p w+q}
\end{gathered}
$$

the nonnegative constants $m, p$, and $q$ satisfy

$$
p>0 \text { and } q>m p,
$$

and the functions $a$ and $b$ satisfy

$a(t)$ and $b(t)$ are nonconstant, nonnegative, nonincreasing, convex,

$-a^{\prime}$ and $-b^{\prime}$ are convex on $[0, \infty)$ and $a(\infty)=b(\infty)=0$.

We consider the question, when is the solution $u$ of $(\dot{i} .1)$ integrable on $[0, \infty)$ uniformly with respect to the parameters $c_{1}, c_{2}$ ? In particular, we ask when do each of the following hold:

$$
\begin{aligned}
& \left.\int_{0}^{\infty} \sup _{0 \leq c_{2} \leq 1}\left|u\left(t, c_{1}, c_{2}\right)\right| d t<\infty \quad \text { (fixed } c_{1} \neq 0\right) \\
& \int_{0}^{\infty} \sup _{0 \leq c_{2} \leq 1}\left|u\left(t, 0, c_{2}\right)\right| d t<\infty \\
& \int_{0}^{\infty} \sup _{0 \leq c_{1} \leq 1}\left|u\left(t, c_{1}, c_{2}\right)\right| d t<\infty \quad\left(\text { fixed } c_{2} \neq 0\right), \\
& \int_{0}^{\infty} \sup _{0 \leq c_{1} \leq 1}\left|u\left(t, c_{1}, 0\right)\right| d t<\infty \\
& \int_{0}^{\infty} \sup _{0 \leq c_{1}, c_{2} \leq 1}\left|u\left(t, c_{1}, c_{2}\right)\right| d t<\infty ?
\end{aligned}
$$

The necessary and (stronger) sufficient conditions below depend on whether $m=0$ or $m \neq 0$. Parts (i) $-(\mathrm{v})$ in Theorem 1.1 give necessary conditions for parts $(\mathrm{i})-(\mathrm{v})$ of (1.6) to hold, respectively.

THEOREM 1.1. Suppose that (1.2)-(1.5) hold. In order that the solution of (1.1) satisfy (1.6) (i) $-(\mathrm{v})$ respectively, it is necessary that the following hold, respectively:

(i) $\quad b \notin L^{1}[0, \infty)$ when $m=0$,

(ii) $\quad a \notin L^{1}[0, \infty)$ or $b \notin L^{1}[0, \infty)$ when $m \neq 0$,

$b \notin L^{1}[0, \infty)$ when $m=0$,

(iii) $\quad a \notin L^{1}[0, \infty)(m \geq 0)$,

(iv) $\quad a \notin L^{1}[0, \infty)(m \geq 0)$,

(v) $\quad a \notin L^{1}[0, \infty)$ when $m \neq 0$,

$a \notin L^{1}[0, \infty)$ and $b \notin L^{1}[0, \infty)$ when $m=0$.

Observe that in part (i) when $m \neq 0$, nothing extra is necessary.

For our sufficient conditions we will use the assumptions
(a) $\int_{1}^{\infty} \frac{\log x}{x A_{a}(x)} d x<\infty$ and
(b) $\int_{1}^{\infty} \frac{\log x}{x A_{b}(x)} d x<\infty$ 
where the functions $A_{a}$ and $A_{b}$ are defined by the formulas

$$
A_{a}(x)=\int_{0}^{x} a(s) d s \text { and } A_{b}(x)=\int_{0}^{x} b(s) d s .
$$

Roughly, the necessary conditions $a \notin L^{1}(0, \infty)$ and $b \notin L^{1}(0, \infty)$ will be replaced by $(1.7)(a)$ and (1.7)(b), respectively, but see the note after Theorem 1.2 in this regard. (Clearly (1.7)(a), (b) imply, respectively, $a \notin L^{1}(0, \infty)$ and $b \notin L^{1}(0, \infty)$ ). If $a(t)=t^{-1} \log ^{q} t, q>1$ (large $\left.t\right)$ or if $a(t)=t^{-p}, 0<p<1$, then (1.7)(a) is satisfied.

THEOREM 1.2. Assume that the conditions (1.2)-(1.5) are satisfied.

(i) Let $c_{1}>0$ be fixed.

(a) If $m \neq 0$ then (1.6)(i) holds.

(b) If $m=0$, then (1.7)(b) implies (1.6)(i).

(ii) Let $c_{1}=0$ be fixed.

(a) If $m \neq 0$, then (1.7)(a) implies (1.6)(ii) holds.

(b) If $m=0$ and (1.7)(a) and (b) are satisfied, then (1.6)(ii) holds.

(iii) Let $c_{2} \neq 0$ be fixed. If (1.7)(a) holds then (1.6)(iii) holds.

(iv) Let $c_{2}=0$ be fixed.

(a) If $m \neq 0$, then (1.7)(a) implies (1.6)(iv).

(b) If $m=0$, assume (1.7)(a) and (b) are satisfied.

Then (1.6)(iv) holds.

(v) (a) If $m \neq 0$ then (1.7)(a) implies (1.6)(v).

(b) If $m=0$, assume $(1.7)(a)$ and (b) are satisfied.

Then $(1.6)(\mathrm{v})$ holds.

Note that in (ii)(a) and (b) we assume that (1.7)(a) holds even though Theorem 1.1 (ii) does not require that $a \notin L^{1}[0, \infty)$ and in (iv)(b) we assume that (1.7)(b) holds even though Theorem 1.1 (iv) does not require that $b \notin L^{1}(0, \infty)$.

The problem (1.1) has been studied in the situation where $m=q=0$ (so that $A(t)$ reduces to $\frac{1}{p}\left(a(t)+c_{1}\right)$ by [4] and [6]. In [4], assuming $a$ satisfies (1.5), it is shown that for the solution $u=u\left(t, c_{1}\right)$ to satisfy

$$
\int_{0}^{\infty} \sup _{0 \leq c_{1} \leq 1}\left|u\left(t, c_{1}\right)\right| d t<\infty
$$

it is necessary that $a \notin L^{1}[0, \infty)$. In [6] it is shown that for $a$ satisfying (1.5) and (1.7)(a), (1.9) holds and in [4] a growth condition at $\infty$ similar to (1.7)(a) is used. Note that $a(0+)<\infty$ is not assumed in [4] and [6].

The form of the function $A$ in (1.2)-(1.5) arises in the study of transverse vibrations in a viscoelastic plate and for longitudinal and bending waves in a viscoelastic

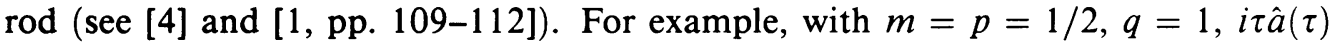


is the complex modulus of shear and $i \tau \hat{b}(\tau)$ is the complex modulus of compression for transverse vibrations in a viscoelastic plate. The tool used is the solution $u_{\lambda}$ of the problem (similar to (1.1), $c_{1}, c_{2}$ fixed)

$$
u_{\lambda}^{\prime}(t)+\lambda \int_{0}^{t} A(t-\tau) u_{\lambda}(\tau) d \tau=0, \quad u_{\lambda}(0)=1, \lambda \geq 1, t \geq 0 .
$$

For results on the question, "When is $\int_{0}^{\infty} \sup _{\lambda \geq 1}\left|u_{\lambda}(t)\right| d t<\infty$ ?", with applications to viscoelasticity, see [3], [5], and when $A(x)=a(x)+c_{1}$ ( $c_{1}$ fixed) see [2].

Two Proofs. We begin with the proof of Theorem 1.1. By [3], we have

$$
\hat{u}\left(\tau, c_{1}, c_{2}\right)=\frac{1}{i \tau+\hat{A}\left(\tau, c_{1}, c_{2}\right)}
$$

and $\hat{u}\left(\tau, c_{1}, c_{2}\right)$ is a continuous function in $\{\operatorname{Im} \tau \leq 0\}$. The argument of [7, pp. 323-324] as arranged in [3] shows that

$$
u\left(\tau, c_{1}, c_{2}\right) \in L^{1}(0, \infty), \quad c_{1}, c_{2} \geq 0 .
$$

Also, from [7], $\hat{a}$ and $\hat{b}$ are differentiable for $\tau>0$ and we have the inequalities

$$
\begin{aligned}
& \frac{1}{2 \sqrt{2}} A_{a}\left(\tau^{-1}\right) \leq|\hat{a}(\tau)| \leq 4 A_{a}\left(\tau^{-1}\right), \quad \tau>0, \\
& \frac{1}{2 \sqrt{2}} A_{b}\left(\tau^{-1}\right) \leq|\hat{b}(\tau)| \leq 4 A_{b}\left(\tau^{-1}\right), \quad \tau>0 .
\end{aligned}
$$

Since $a(\infty)=b(\infty)=0$, we have

$$
\lim _{x \rightarrow \infty} \frac{x}{A_{a}(x)+A_{b}(x)}=\infty .
$$

It follows from this and from (2.1) and (2.2) that

$$
\begin{aligned}
\int_{0}^{\infty} u\left(t, c_{1}, c_{2}\right) d t & =\lim _{\tau \rightarrow 0+} \hat{u}\left(\tau, c_{1}, c_{2}\right)=\lim _{\tau \rightarrow 0+} \frac{1}{i \tau+\hat{A}\left(\tau, c_{1}, c_{2}\right)} \\
& =\lim _{\tau \rightarrow 0+} \frac{p \hat{b}(\tau)+q \hat{a}(\tau)-i \tau^{-1}\left(p c_{2}+q c_{1}\right)}{\left(\hat{a}(\tau)-i \tau^{-1} c_{1}\right)\left(\hat{b}(\tau)+m \hat{a}(\tau)-i \tau^{-1}\left(c_{2}+m c_{1}\right)\right)} .
\end{aligned}
$$

If we denote $\|f\| \equiv \int_{0}^{\infty} f(t) d t$ for $f=a$ or $f=b$ and if $q /\|b\|$ and $p /\|a\|$ are understood to be zero when $b \notin L^{1}(0, \infty)$, respectively $a \notin L^{1}(0, \infty)$, we use (2.3) and (2.4) to obtain

$$
\begin{aligned}
\int_{0}^{\infty} u\left(t, c_{1}, c_{2}\right) d t=0, & \text { if } c_{1} \neq 0, c_{2} \neq 0, \\
0, & \text { if } c_{1} \neq 0, c_{2}=0, m \neq 0, \\
q /\|b\|, & \text { if } c_{1} \neq 0, c_{2}=0, m=0, \\
p /\|a\|, & \text { if } c_{1}=0, c_{2} \neq 0, \\
\frac{p\|b\|+q\|a\|}{\|a\|(\|b\|+m\|a\|)}, & \text { if } c_{1}=0, c_{2}=0, a, b \in L^{1}, \\
p /\|a\|, & \text { if } c_{1}=0, c_{2}=0, b \notin L^{1}, a \in L^{1}, \\
0, & \text { if } c_{1}=0, c_{2}=0, b \in L^{1}, a \notin L^{1}, m \neq 0, \\
q /\|b\|, & \text { if } c_{1}=0, c_{2}=0, b \in L^{1}, a \notin L^{1}, m=0, \\
0, & \text { if } c_{1}=0, c_{2}=0, a \notin L^{1}, b \notin L^{1},
\end{aligned}
$$


where $L^{1}=L^{1}(0, \infty)$. Since $(2.5)$ implies that

$$
\begin{aligned}
\int_{0}^{\infty}\left|u\left(t, c_{1}, c_{2}\right)-u\left(t, c_{1}, 0\right)\right| d t & \geq \lim _{\tau \rightarrow 0+}\left|\hat{u}\left(\tau, c_{1}, c_{2}\right)-\hat{u}\left(\tau, c_{1}, 0\right)\right| \\
& =\left|\int_{0}^{\infty} u\left(t, c_{1}, c_{2}\right) d t-\int_{0}^{\infty} u\left(t, c_{1}, 0\right) d t\right| \\
& =|0-q /\|b\||>0,
\end{aligned}
$$

when $c_{1}, c_{2} \neq 0, m=0$, we see that a necessary condition for (1.6)(i) to hold when $m=0$ is that $b \notin L^{1}(0, \infty)$. This proves Theorem 1.1(i). This procedure is also used to prove parts (ii) $-(\mathrm{v})$ of Theorem 1.1.

Next we will prove Theorem 1.2. We start with the representation

$$
\pi u\left(t, c_{1}, c_{2}\right)=-\operatorname{Im}\left\{\frac{1}{t} \int_{0}^{\infty} e^{i \tau t} \frac{D_{\tau}\left(\tau, c_{1}, c_{2}\right)}{D\left(\tau, c_{1}, c_{2}\right)^{2}} d \tau\right\}, \quad t>0,
$$

established in $[3,(2.23)$ and $4,(4.32)]$ (and valid here even though $-a^{\prime}(0+)-b^{\prime}(0+)=$ $\infty$ is allowed), where

$$
\begin{aligned}
D\left(\tau, c_{1}, c_{2}\right) & \equiv \frac{\left(\hat{a}(\tau)-i \tau^{-1} c_{1}\right)\left(\hat{b}(\tau)+m \hat{a}(\tau)-i \tau^{-1}\left(c_{2}+m c_{1}\right)\right)}{p \hat{b}(\tau)+q \hat{a}(\tau)-i \tau^{-1}\left(p c_{2}+q c_{1}\right)}+i \tau \\
& \equiv \frac{F_{1} F_{2}}{F_{3}}+i \tau
\end{aligned}
$$

Also, by $[3,(1.15)]$,

$$
\left|u\left(t, c_{1}, c_{2}\right)\right| \leq 1, \quad t \geq 0,
$$

so we only need to obtain an upper bound for $(2.6)$ on $[L, \infty)$ (for some $L>0$ ) in order to establish (1.6). In each part of Theorem 1.2, we follow the same procedure to establish the inequality $\left|u\left(t, c_{1}, c_{2}\right)\right| \leq M f(t)$ where the function $f$, defined in the last paragraph of the paper satisfies $\int_{L}^{\infty} f(t) d t<\infty$ and $M$ is a constant independent of the parameter (parameters) that is (are) allowed to vary. Let us look at the path we will take in our proof and point out the terms that will need to be estimated in each part.

Since $D_{\tau}\left(\tau, c_{1}, c_{2}\right)=\left(F_{1} F_{2} / F_{3}\right)^{\prime}+i$, we have, by $(2.6)$,

$$
\left|\pi u\left(t, c_{1}, c_{2}\right)\right| \leq\left|T_{1}\right|+\left|T_{2}\right|,
$$

where

$$
T_{1}=\frac{1}{t} \int_{0}^{\infty} e^{i \tau t}\left(\frac{F_{1} F_{2}}{F_{3}}\right)^{\prime} / D\left(\tau, c_{1}, c_{2}\right)^{2} d \tau
$$

and

$$
T_{2}=\frac{1}{t} \int_{0}^{\infty} e^{i \tau t} / D\left(\tau, c_{1}, c_{2}\right)^{2} d \tau
$$

By the triangle inequality,

$$
\begin{aligned}
\left|T_{2}\right| & \leq \frac{1}{t} \int_{0}^{1 / t} \frac{d \tau}{\left|D\left(\tau, c_{1}, c_{2}\right)\right|^{2}}+\frac{1}{t}\left|\int_{1 / t}^{\infty} \frac{e^{i \tau t}}{D\left(\tau, c_{1}, c_{2}\right)^{2}} d \tau\right| \\
& \equiv T_{2,1}+T_{2,2} .
\end{aligned}
$$


Thus, to obtain an upper bound for $\left|T_{2}\right|$ we will estimate

$$
T_{2,1}=\frac{1}{t} \int_{0}^{1 / t} \frac{d \tau}{\left|D\left(\tau, c_{1}, c_{2}\right)\right|^{2}}
$$

and the right-hand side of the inequality

$$
T_{2,2} \leq \frac{2}{t^{2}}\left(\frac{1}{\left|D\left(1 / t, c_{1}, c_{2}\right)\right|^{2}}+\int_{1 / t}^{\infty}\left[\left|\left(\frac{F_{1} F_{2}}{F_{3}}\right)^{\prime}\right|+1\right] \frac{d \tau}{\left|D\left(\tau, c_{1}, c_{2}\right)\right|^{3}}\right)
$$

where the inequality in (2.11) follows from an integration by parts. The estimates below in the proofs of the different parts of Theorem 1.2 assure the absolute convergence of the integral as well as the vanishing of the boundary term at $\infty$.

For upper bounds on $T_{1}$ we write

$$
T_{1}=\frac{1}{t} \int_{0}^{1 / t}+\frac{1}{t} \int_{1 / t}^{\infty} \equiv T_{1,1}+T_{1,2} .
$$

We will estimate

$$
\left|T_{1,1}\right|=\left|\frac{1}{t} \int_{0}^{1 / t} e^{i \tau t}\left(\frac{F_{1} F_{2}}{F_{3}}\right)^{\prime} / D\left(\tau, c_{1}, c_{2}\right)^{2} d \tau\right|
$$

and the right side of

$$
\begin{aligned}
\left|T_{1,2}\right| \leq & \frac{1}{t^{2}}\left|\left(\frac{F_{1} F_{2}}{F_{3}}\right)_{\tau=t^{-1}}^{\prime} \frac{1}{D\left(t^{-1}, c_{1}, c_{2}\right)^{2}}\right| \\
& +\frac{2}{t^{2}} \int_{1 / t}^{\infty}\left(\left|\left(\frac{F_{1} F_{2}}{F_{3}}\right)^{\prime \prime} / D\left(\tau, c_{1}, c_{2}\right)^{2}\right|\right. \\
& +\left|\left(\frac{F_{1} F_{2}}{F_{3}}\right)^{\prime^{2}} / D\left(\tau, c_{1}, c_{2}\right)^{3}\right| \\
& \left.+\left|\left(\frac{F_{1} F_{2}}{F_{3}}\right)^{\prime} / D\left(\tau, c_{1}, c_{2}\right)^{3}\right|\right) d \tau \equiv B+I,
\end{aligned}
$$

where the inequality in (2.13) follows by an integration by parts and the estimates below assure the absolute convergence of the integral and the vanishing of the boundary term at $\infty$.

We will show that $\left|T_{1,1}\right|+\left|T_{1,2}\right|+\left|T_{2,1}\right|+\left|T_{2,2}\right| \leq M f(t) \in L^{1}(L, \infty)$, where $M$ is independent of the parameter(s) we are allowing to vary. Then (2.8) and (2.9) will show that the corresponding part of (1.6) holds. To make the needed estimates in (2.10)-(2.13) we will choose constants $\varepsilon$ (small) and $K$ (large) and use upper bounds on the functions $F_{i}^{(j)}(\tau), i=1,2,3, j=0,1,2$ (defined in (2.7)) for $\tau$ in the intervals $[0, \varepsilon],[\varepsilon, K]$, and $[K, \infty]$. We will also need lower bounds for the functions $F_{i}(\tau)$, $i=1,2,3$, and $D\left(\tau, c_{1}, c_{2}\right)$ for $0 \leq \tau \leq \varepsilon$ and for the functions $F_{3}(\tau), D\left(\tau, c_{1}, c_{2}\right)$ on $[\varepsilon, K]$ and $[K, \infty]$. 
We will use the following lower bounds (which follow easily from (2.7)) in each part of Theorem 1.2:

(i) $\left|F_{1}\right| \geq \max \left\{|\hat{a}(\tau)|, \tau^{-1} c_{1}\right\}, \quad \tau>0$,

(ii) $\left|F_{2}\right| \geq \max \left\{|\hat{b}(\tau)|, m|\hat{a}(\tau)|, \tau^{-1}\left(m c_{1}+c_{2}\right)\right\}, \quad \tau>0$,

(iii) $\left|F_{3}\right| \geq \max \left\{p|\hat{b}(\tau)|, q|\hat{a}(\tau)|, \tau^{-1}\left(q c_{1}+p c_{2}\right)\right\}, \quad \tau>0$.

We use the inequalities (see (1.8))

$$
\infty \geq \lim _{x \rightarrow 0+} \frac{A_{a}(x)}{x}=a(0+)>0 \text { and } \infty \geq \lim _{x \rightarrow 0+} \frac{A_{b}(x)}{x}=b(0+)>0,
$$

and (2.4) to obtain the existence of positive constants $M_{1}, \delta_{1}$, and $K_{1}$ so that

(i) $\quad M_{1} A_{a}(x) \geq x, \quad M_{1} A_{b}(x) \geq x, \quad 0 \leq x \leq \delta_{1}$,

(ii) $A_{a}(x) \leq M_{1} x, \quad A_{b}(x) \leq M_{1} x, \quad K_{1} \leq x$.

Since the proofs of all the different parts of Theorem 1.2 are so similar, we will prove (iv)(a) and omit the other proofs. For the rest of the paper we let $M$ be a constant whose exact value may change each time that it appears.

Proof of Theorem 1.2 (iv)(a).

We will be using the inequalities

$$
\begin{aligned}
& \left|\hat{a}^{\prime}(\tau)\right| \leq M \int_{0}^{1 / \tau} s a(s) d s \leq M \tau^{-1} A_{a}\left(\tau^{-1}\right), \\
& \left|\hat{b}^{\prime}(\tau)\right| \leq M \int_{0}^{1 / \tau} s b(s) d s \leq M \tau^{-1} A_{b}\left(\tau^{-1}\right), \quad \tau>0,
\end{aligned}
$$

and

$$
\begin{aligned}
& \left|\hat{a}^{\prime \prime}(\tau)\right| \leq M \int_{0}^{1 / \tau} s^{2} a(s) d s \leq M \tau^{-2} A_{a}\left(\tau^{-1}\right), \\
& \left|\hat{b}^{\prime \prime}(\tau)\right| \leq M \int_{0}^{1 / \tau} s^{2} b(s) d s \leq M \tau^{-2} A_{b}\left(\tau^{-1}\right), \quad \tau>0,
\end{aligned}
$$

from [2, (4.2) and (5.3)]. (It is for formula (2.17) that the assumptions that $-a^{\prime}$ and $-b^{\prime}$ are convex are used).

From the definition of $F_{1}, F_{2}$, and $F_{3}$, we use (2.3), (2.16), and (2.17) to obtain $M>0$ and $\varepsilon>0$ such that the following hold:

$$
\begin{aligned}
& \text { (i) } \quad\left|F_{1}(\tau)\right| \leq M \max \left\{A_{a}\left(\tau^{-1}\right), c_{1} \tau^{-1}\right\}, \\
&\left|F_{2}(\tau)\right| \leq M \max \left\{A_{b}\left(\tau^{-1}\right), A_{a}\left(\tau^{-1}\right), c_{1} \tau^{-1}\right\}, \\
&\left|F_{3}(\tau)\right| \leq M \max \left\{A_{b}\left(\tau^{-1}\right), A_{a}\left(\tau^{-1}\right), c_{1} \tau^{-1}\right\}, \\
&\left|F_{1}^{\prime}(\tau)\right| \leq M \tau^{-1} \max \left\{A_{a}\left(\tau^{-1}\right), c_{1} \tau^{-1}\right\}, \\
&\left|F_{2}^{\prime}(\tau)\right| \leq M \tau^{-1} \max \left\{A_{b}\left(\tau^{-1}\right), A_{a}\left(\tau^{-1}\right), c_{1} \tau^{-1}\right\}, \\
&\left|F_{3}^{\prime}(\tau)\right| \leq M \tau^{-1} \max \left\{A_{b}\left(\tau^{-1}\right), A_{a}\left(\tau^{-1}\right), c_{1} \tau^{-1}\right\}, \quad 0<\tau \leq \varepsilon, \\
& \text { (iii) } \quad\left|F_{1}^{\prime \prime}(\tau)\right| \leq M \tau^{-2} \max \left\{A_{a}\left(\tau^{-1}\right), c_{1} \tau^{-1}\right\}, \\
&\left|F_{2}^{\prime \prime}(\tau)\right| \leq M \tau^{-2} \max \left\{A_{b}\left(\tau^{-1}\right), A_{a}\left(\tau^{-1}\right) c_{1} \tau^{-1}\right\}, \quad \\
&\left|F_{3}^{\prime \prime}(\tau)\right| \leq M \tau^{-2} \max \left\{A_{b}\left(\tau^{-1}\right), A_{a}\left(\tau^{-1}\right), c_{1} \tau^{-1}\right\}, \quad 0<\tau \leq \varepsilon,
\end{aligned}
$$


and

$$
\begin{aligned}
& M\left|F_{1}(\tau)\right| \geq \max \left\{A_{a}\left(\tau^{-1}\right), c_{1} \tau^{-1}\right\}, \\
& M\left|F_{2}(\tau)\right| \geq \max \left\{A_{b}\left(\tau^{-1}\right), A_{a}\left(\tau^{-1}\right), c_{1} \tau^{-1}\right\}, \\
& M\left|F_{3}(\tau)\right| \geq \max \left\{A_{b}\left(\tau^{-1}\right), A_{a}\left(\tau^{-1}\right), c_{1} \tau^{-1}\right\}, \quad 0<\tau \leq \varepsilon .
\end{aligned}
$$

Making $\varepsilon$ smaller, if necessary, we have

$$
\begin{aligned}
\left|D\left(\tau, c_{1}, 0\right)\right| & \geq\left|\frac{F_{1} F_{2}}{F_{3}}\right|-\tau \\
& \geq \frac{\max \left\{A_{a}\left(\tau^{-1}\right), c_{1} \tau^{-1}\right\} \max \left\{A_{b}\left(\tau^{-1}\right), A_{a}\left(\tau^{-1}\right), c_{1} \tau^{-1}\right\}}{M \max \left\{A_{b}\left(\tau^{-1}\right), A_{a}\left(\tau^{-1}\right), c_{1} \tau^{-1}\right\}}-\tau \\
& \geq \frac{1}{M} \max \left\{A_{a}\left(\tau^{-1}\right), c_{1} \tau^{-1}\right\}, \quad 0<\tau \leq \varepsilon,
\end{aligned}
$$

where we have used (2.18) and (2.19).

Next we use (2.15)(i) and the definition of $F_{1}, F_{2}$, and $F_{3}$ to obtain $M>0$ and $K>0$ such that

(i) $\left|F_{1}(\tau)\right| \leq M A_{a}\left(\tau^{-1}\right), \quad\left|F_{2}(\tau)\right| \leq M\left(A_{b}\left(\tau^{-1}\right)+A_{a}\left(\tau^{-1}\right)\right)$,

$$
\left|F_{3}(\tau)\right| \leq M\left(A_{b}\left(\tau^{-1}\right)+A_{a}\left(\tau^{-1}\right)\right), \quad K \leq \tau,
$$

(ii) $\quad\left|F_{1}^{\prime}(\tau)\right| \leq M \tau^{-1} A_{a}\left(\tau^{-1}\right), \quad\left|F_{2}^{\prime}(\tau)\right| \leq M \tau^{-1}\left(A_{b}\left(\tau^{-1}\right)+A_{a}\left(\tau^{-1}\right)\right)$,

$$
\left|F_{3}^{\prime}(\tau)\right| \leq M \tau^{-1}\left(A_{b}\left(\tau^{-1}\right)+A_{a}\left(\tau^{-1}\right)\right), \quad K \leq \tau,
$$

(iii) $\left|F_{1}^{\prime \prime}(\tau)\right| \leq M \tau^{-2} A_{a}\left(\tau^{-1}\right), \quad\left|F_{2}^{\prime \prime}(\tau)\right| \leq M \tau^{-2}\left(A_{b}\left(\tau^{-1}\right)+A_{a}\left(\tau^{-1}\right)\right)$,

$$
\left|F_{3}^{\prime \prime}(\tau)\right| \leq M \tau^{-2}\left(A_{b}\left(\tau^{-1}\right)+A_{a}\left(\tau^{-1}\right)\right), \quad K \leq \tau,
$$

and

$$
\left|F_{3}(\tau)\right| \geq \frac{1}{M}\left(A_{b}\left(\tau^{-1}\right)+A_{a}\left(\tau^{-1}\right)\right), \quad K \leq \tau .
$$

By (2.21) and (2.22) we also have (make $K$ larger if necessary)

$$
\begin{aligned}
\left|D\left(\tau, c_{1}, 0\right) \geq \tau-\right| \frac{F_{1} F_{2}}{F_{3}} \mid & \geq \tau-\frac{M A_{a}\left(\tau^{-1}\right)\left(A_{b}\left(\tau^{-1}\right)+A_{a}\left(\tau^{-1}\right)\right)}{A_{b}\left(\tau^{-1}\right)+A_{a}\left(\tau^{-1}\right)} \\
& \geq \frac{\tau}{2}, \quad K \leq \tau .
\end{aligned}
$$

Because the real part of $\hat{a}(\tau)(\operatorname{Re} \hat{a}(\tau))$ is a continuous function on $\tau>0$ (see [2]),

$$
\frac{1}{M} \leq\left|F_{i}^{(j)}(\tau)\right| \leq M, \quad \varepsilon \leq \tau \leq K, \quad \text { for } i=1,2,3, j=0,1,2,
$$

and

$$
\left|D\left(\tau, c_{1}, 0\right)\right| \geq \frac{1}{M}, \quad \varepsilon \leq \tau \leq K,
$$

are obviously satisfied.

Because $\left|u\left(t, c_{1}, c_{2}\right)\right| \leq 1, t \geq 0$, we only need to estimate (2.10)-(2.13) for $t \geq \frac{1}{\varepsilon}$. Let $t \geq \frac{1}{\varepsilon}$. We begin with (2.10). By (2.20) we have

$$
T_{2,1}=\frac{1}{t} \int_{0}^{1 / t} \frac{d \tau}{\left|D\left(\tau, c_{1}, 0\right)\right|^{2}} \leq \frac{M}{t} \int_{0}^{1 / t} \frac{d \tau}{A_{a}^{2}\left(\tau^{-1}\right)} \leq \frac{M}{t^{2} A_{a}^{2}\left(\varepsilon^{-1}\right)} \leq \frac{M}{t^{2}}
$$


Now we estimate the boundary term in (2.11). By (2.20), we have

$$
\frac{2}{t^{2}} \frac{1}{\left|D\left(1 / t, c_{1}, 0\right)\right|} \leq \frac{M}{t^{2} A_{a}^{2}(t)} \leq \frac{M}{t^{2}} .
$$

We write the integral term in $(2.11)$ as

Because

$$
\frac{1}{t^{2}}\left(\int_{1 / t}^{\varepsilon}+\int_{\varepsilon}^{K}+\int_{K}^{\infty}\right) \frac{1}{\left|D\left(\tau, c_{1}, 0\right)\right|^{3}}\left(\left(\frac{F_{1} F_{2}}{F_{3}}\right)^{\prime}+1\right) d \tau
$$

$$
\left(\frac{F_{1} F_{2}}{F_{3}}\right)^{\prime}=\frac{F_{1}^{\prime} F_{2}}{F_{3}}+\frac{F_{1} F_{2}^{\prime}}{F_{3}}-\frac{F_{1} F_{2} F_{3}^{\prime}}{F_{3}^{2}}
$$

we use (2.18)(i) and (ii), (2.19), and (2.20) to estimate the first integral in (2.26). Thus, we have

$$
\begin{aligned}
\frac{1}{t^{2}} \int_{1 / t}^{\varepsilon} & \leq \frac{M}{t^{2}} \int_{1 / t}^{\varepsilon} \frac{1}{\max \left\{A_{a}\left(\tau^{-1}\right), c_{1} \tau^{-1}\right\}^{3}}\left[\tau^{-1} \max \left\{A_{a}\left(\tau^{-1}\right), c_{1} \tau^{-1}\right\}+1\right] d \tau \\
& \leq \frac{M}{t^{2}} \int_{1 / t}^{\varepsilon} \frac{\tau^{-1}}{A_{a}^{2}\left(\tau^{-1}\right)} d \tau \leq \frac{M}{t^{2}} \int_{1 / t}^{\varepsilon} \tau^{-1} d \tau=\frac{M \log \varepsilon t}{t^{2}},
\end{aligned}
$$

where the second inequality also uses the fact that $\tau / A_{a}\left(\tau^{-1}\right)$ is a bounded function on $0<\tau \leq \varepsilon$. By (2.24) and (2.25), it follows that

$$
\frac{1}{t^{2}} \int_{\varepsilon}^{K} \leq \frac{M}{t^{2}}
$$

By (2.21)(i), (ii), (2.22), and (2.23), we have

$$
\frac{1}{t^{2}} \int_{K}^{\infty} \leq \frac{M}{t^{2}} \int_{K}^{\infty} \frac{1}{\tau^{3}}\left[\tau^{-1} A_{a}\left(\tau^{-1}\right)+1\right] d \tau \leq \frac{M}{t^{2}} .
$$

Thus we have shown (see (2.11)) that

$$
T_{2,2} \leq \frac{M \log \varepsilon t}{t^{2}}+\frac{M}{t^{2}}, \quad t \geq 1 / \varepsilon
$$

Now we move to (2.12). For this estimate we use (2.18)(i), (ii), (2.19), and (2.20) to obtain

$$
\begin{aligned}
& \frac{1}{t} \int_{0}^{1 / t} \frac{1}{\left|D\left(\tau, c_{1}, 0\right)\right|^{2}}\left|\left(\frac{F_{1} F_{2}}{F_{3}}\right)^{\prime}\right| d \tau \\
& \quad \leq \frac{M}{t} \int_{0}^{1 / t} \frac{\tau^{-1} \max \left\{A_{a}\left(\tau^{-1}\right), c_{1} \tau^{-1}\right\}}{\max \left\{A_{a}\left(\tau^{-1}\right), c_{1} \tau^{-1}\right\}^{2}} d \tau \\
& \quad \leq \frac{M}{t} \int_{0}^{1 / t} \frac{\tau^{-1}}{A_{a}\left(\tau^{-1}\right)} d \tau \equiv f_{1}(t) .
\end{aligned}
$$

The assumption (1.7)(a) and the Fubini theorem show that

$$
\begin{aligned}
\int_{1 / \varepsilon}^{\infty} f_{1}(t) d t & =M \int_{0}^{\varepsilon} \frac{\tau^{-1}}{A_{a}\left(\tau^{-1}\right)} \int_{1 / \varepsilon}^{1 / \tau} \frac{1}{t} d t d \tau \\
& =M \int_{0}^{\varepsilon} \frac{\tau^{-1} \log \left(\varepsilon \tau^{-1}\right)}{A_{a}\left(\tau^{-1}\right)} d \tau \\
& =M \int_{1 / \varepsilon}^{\infty} \frac{\log \varepsilon x}{x A_{a}(x)} d x<\infty
\end{aligned}
$$


Lastly we will estimate the terms in (2.13). By (2.18)(i), (ii), (2.19), and (2.20) we see that

$$
B \leq \frac{M}{t^{2}}\left(\frac{t}{A(t)}\right)=\frac{M}{t A(t)}=f_{2}(t),
$$

and $f_{2}(t) \in L^{1}(1 / \varepsilon, \infty)$ by $(1.7)(\mathrm{a})$. The term $I$ can be written as

$$
\frac{2}{t^{2}}\left[\int_{1 / t}^{\varepsilon}+\int_{\varepsilon}^{K}+\int_{K}^{\infty}\right] V d \tau,
$$

where

$$
V \equiv\left(\frac{\left|\left(\frac{F_{1} F_{2}}{F_{3}}\right)^{\prime \prime}\right|}{\left|D\left(\tau, c_{1}, 0\right)\right|^{2}}+\frac{\left|\left(\frac{F_{1} F_{2}}{F_{3}}\right)^{\prime^{2}}\right|}{\left|D\left(\tau, c_{1}, 0\right)\right|^{3}}+\frac{\left|\left(\frac{F_{1} F_{2}}{F_{3}}\right)^{\prime}\right|}{\left|D\left(\tau, c_{1}, 0\right)\right|^{3}}\right) \text {. }
$$

By writing out

$$
\left(\frac{F_{1} F_{2}}{F_{3}}\right)^{\prime \prime}=\frac{F_{1}^{\prime \prime} F_{2}}{F_{3}}+\frac{2 F_{1}^{\prime} F_{2}^{\prime}}{F_{3}}+\text { five more terms, }
$$

and using

$$
\left(\frac{F_{1} F_{2}}{F_{3}}\right)^{\prime}=\frac{F_{1}^{\prime} F_{2}}{F_{3}}+\text { two more terms, }
$$

we can use (2.18)(i)-(iii) and (2.19)-(2.25) to make the required estimates. Thus by $(2.18)-(2.20)$ we have

$$
\begin{aligned}
\frac{2}{t^{2}} \int_{1 / t}^{\varepsilon} & \leq \frac{M}{t^{2}} \int_{1 / t}^{\varepsilon} \frac{\tau^{-2}}{A_{a}\left(\tau^{-1}\right)}+\frac{\tau^{-2}}{A_{a}\left(\tau^{-1}\right)}+\frac{\tau^{-1}}{A_{a}^{2}\left(\tau^{-1}\right)} d \tau \\
& \leq \frac{M}{t^{2}} \int_{1 / t}^{\varepsilon} \frac{\tau^{-2}}{A_{a}\left(\tau^{-1}\right)} d \tau \equiv f_{3}(t),
\end{aligned}
$$

where $f_{3}(t) \in L^{1}(1 / \varepsilon, \infty)$ by $(1.7)(\mathrm{a})$. By $(2.24)$ and (2.25) we also have

$$
\frac{2}{t^{2}} \int_{1 / t}^{\varepsilon} \leq \frac{M}{t^{2}}
$$

Finally, by $(2.21)-(2.25)$ we have

$$
\frac{2}{t^{2}} \int_{\varepsilon}^{K} \leq \frac{M}{t^{2}}
$$

With the estimates on (2.10)-(2.13) made above, we also recall that

$$
\left|\pi u\left(t, c_{1}, 0\right)\right| \leq\left|T_{1}\right|+\left|T_{2}\right| \leq\left|T_{1,1}\right|+\left|T_{1,2}\right|+T_{2,1}+T_{2,2}
$$

Thus we have

$$
\left|u\left(t, c_{1}, 0\right)\right| \leq M\left(\frac{\log \varepsilon t}{t^{2}}+\frac{1}{t^{2}}+f_{1}(t)+f_{2}(t)+f_{3}(t)\right) \equiv f(t), \quad t \geq 1 / \varepsilon,
$$

where $f \in L^{1}(1 / \varepsilon, \infty)$. With the fact, already mentioned, that $\left|u\left(t, c_{1}, 0\right)\right| \leq 1, t \geq 0$, the proof that (1.6)(iv) holds is complete. 


\section{REFERENCES}

[1] D. R. Bland, The theory of linear viscoelasticity, Pergamon Press, New York, 1960

[2] R. W. Carr and K. B. Hannsgen, A nonhomogeneous integrodifferential equation in Hilbert space, SIAM J. Math. Anal. 10, 961-984 (1979)

[3] K. B. Hannsgen, A linear integrodifferential equation for viscoelastic rods and plates, Quart. Appl. Math. 41, 75-83 (1983)

[4] K. B. Hannsgen and R. L. Wheeler, $A$ singular limit problem for an integrodifferential equation, J. of Integral Equations 5, 199-209 (1983)

[5] R. D. Noren, A linear Volterra integrodifferential equation for viscoelastic rods and plates, Quart. Appl. Math. 45, 503-514 (1987)

[6] R. D. Noren, $A$ singular limit problem for a Volterra equation, SIAM J. Math. Anal., to appear

[7] D. F. Shea and S. Wainger, Variants of the Wiener-Levy theorem, with applications to stability problems for some Volterra integral equations, Amer. J. Math. 97, 312-343 (1975) 